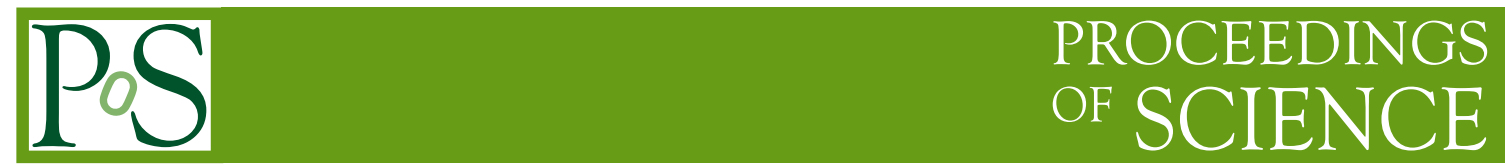

\title{
CP-violating Axions
}

\section{Luca Di Luzio ${ }^{a, *}$}

${ }^{a}$ Dipartimento di Fisica e Astronomia 'G. Galilei', Università di Padova, Italy Istituto Nazionale Fisica Nucleare, Sezione di Padova, Italy

E-mail: luca.diluzio@unipd.it

While the axion was originally introduced to "wash out" CP violation from strong interactions, new sources of CP violation beyond QCD might manifest themselves via a tiny scalar axion-nucleon component. The latter can be experimentally probed in axion-mediated force experiments, as suggested long ago by J.E. Moody and F. Wilczek. In the present note, I review the physical origin of CP-violating axion couplings and point out the special role of the QCD axion as a low-energy portal to high-energy sources of $\mathrm{CP}$ violation.

The European Physical Society Conference on High Energy Physics (EPS-HEP2021), 26-30 July 2021

Online conference, jointly organized by Universität Hamburg and the research center DESY

\footnotetext{
${ }^{*}$ Speaker
} 


\section{QCD axion \& $\mathrm{CP}$}

The QCD axion originally emerged from the need to "wash out" CP violation from strong interactions [1-4]. From an effective field theory perspective, the Peccei-Quinn (PQ) solution to the strong CP problem can be formulated as follows. The SM Lagrangian is augmented by a spin- 0 field $a(x)$ endowed with a pseudo-shift symmetry $a \rightarrow a+\alpha f_{a}$, that is broken only by the operator $\frac{a}{f_{a}} \frac{\alpha_{s}}{8 \pi} G \tilde{G}$, with $G \tilde{G} \equiv \frac{1}{2} \epsilon^{\mu \nu \rho \sigma} G_{\mu \nu}^{a} G_{\rho \sigma}^{a}$. After employing the pseudo-shift symmetry to reabsorb the QCD $\theta$ term by setting $\alpha=-\theta$, one is left in the shifted theory with the $a G \tilde{G}$ operator. Hence, the question of $\mathrm{CP}$ violation in strong interactions is traded for a dynamical question about the vacuum expectation value (VEV) of the axion field $\theta_{\text {eff }} \equiv \frac{\langle a\rangle}{f_{a}}$, resulting into an effective $\theta$ parameter, with $\left|\theta_{\mathrm{eff}}\right| \lesssim 10^{-10}$ from the non-observation of the neutron electric dipol moment (nEDM).

A general result, due to Vafa and Witten [5] ensures that the ground state energy density of QCD $\mathscr{E}$ is minimized for $\theta_{\text {eff }}=0$, namely $\mathscr{E}(0) \leq \mathscr{E}\left(\theta_{\text {eff }}\right)$. The argument is based on an inequality that exploits the path-integral representation of $\mathscr{E}$. A crucial assumption, on which the proof relies on, consists in the positive definiteness of the path-integral measure. However, while the fermionic determinant is positive definite in vector-like theory like QCD that is not the case for a chiral theory like the Standard Model (SM). Hence, we cannot apply the the Vafa-Witten theorem to the SM, although the argument does not automatically imply that $\theta_{\text {eff }} \neq 0$ in the SM.

An extra ingredient of the SM is that CP is explicitly broken in the quark sector by the Cabibbo-Kobayashi-Maskawa (CKM) phase, which sources a $\theta_{\text {eff }} \neq 0$ term. Given a shortdistance CP-violating local operator $\mathcal{O}_{\mathrm{CPV}}(x)$, we can estimate the value of $\theta_{\text {eff }}$ by expanding the axion potential as $V\left(\theta_{\mathrm{eff}}\right)=K^{\prime} \theta_{\mathrm{eff}}+\frac{1}{2} K \theta_{\mathrm{eff}}^{2}+O\left(\theta_{\mathrm{eff}}^{3}\right)$, and focus on the $\theta_{\mathrm{eff}} \ll 1$ regime. Here, $K=i \int d^{4} x\left\langle 0\left|T \frac{\alpha_{s}}{8 \pi} G \tilde{G}(x) \frac{\alpha_{s}}{8 \pi} G \tilde{G}(0)\right| 0\right\rangle$ is a 2-point function also known as topological susceptibility, while $K^{\prime}=i \int d^{4} x\left\langle 0\left|T \frac{\alpha_{s}}{8 \pi} G \tilde{G}(x) \mathcal{O}_{\mathrm{CPV}}(0)\right| 0\right\rangle$. The induced $\theta_{\mathrm{eff}}$ is hence obtained by the direct minimization of $V\left(\theta_{\mathrm{eff}}\right)$, yielding $\theta_{\mathrm{eff}} \simeq-\frac{K^{\prime}}{K}$.

\section{2. $\theta_{\text {eff }}$ in the Standard Model}

The value of $\theta_{\text {eff }}$ in the SM was estimated by Georgi and Randall in Ref. [6]. At energies below $\Lambda_{\chi}=4 \pi F_{\pi}$ with $F_{\pi} \simeq 92 \mathrm{MeV}$ one can write a flavour conserving, CP-violating operator $\mathcal{O}_{\mathrm{CPV}}^{\mathrm{SM}}=\frac{G_{F}^{2}}{m_{c}^{2}} J_{\mathrm{CKM}}\left[\bar{u} \gamma^{\mu}\left(1-\gamma_{5}\right) d \cdot \bar{d} \gamma_{\mu}\right] \not D\left[\gamma^{v}\left(1-\gamma_{5}\right) s \cdot \bar{s} \gamma_{v}\left(1-\gamma_{5}\right) u\right]$, which is obtained in the SM after integrating out the $W$ boson and the charm quark (see diagram in Fig. 1 of [6]), while $J_{\mathrm{CKM}}=\operatorname{Im} V_{u d} V_{c d}^{*} V_{c s} V_{u s}^{*} \simeq 3 \times 10^{-5}$ is the reduced Jarlskog invariant. A proper evaluation of the $K^{\prime}$ matrix element in the presence of $\mathcal{O}_{\mathrm{CPV}}^{\mathrm{SM}}$ is far from trivial. However, by the rules of naive dimensional analysis (NDA) one expects $K^{\prime} \sim F_{\pi}^{4} \frac{G_{F}^{2}}{m_{c}^{2}} J_{\mathrm{CKM}} F_{\pi}^{4} \Lambda_{\chi}^{2}$ [6]. Hence, taking also $K \sim F_{\pi}^{4}$, one obtains the estimate

$$
\theta_{\mathrm{eff}}^{\mathrm{SM}} \sim \frac{G_{F}^{2}}{m_{c}^{2}} J_{\mathrm{CKM}} F_{\pi}^{4} \Lambda_{\chi}^{2} \sim 10^{-19},
$$

which should be taken only as indicative, since it could be off also at the order of magnitude level. Anyway, the estimate in Eq. (1) shows that the PQ mechanism works in the SM also thanks to the SM inner structure. Indeed, the CKM contribution to $\theta_{\mathrm{eff}}^{\mathrm{SM}}$ could have easily overshoot $10^{-10}$ if the QCD and the Fermi scale would have been closer and/or in the presence of a trivial flavour structure such that $J_{\mathrm{CKM}} \sim 1$. Also, the PQ mechanism is not generically going to work in a low-scale 
theory beyond the SM with generic $\mathrm{CP}$ violation. To see this, assume a $d=6 \mathrm{CP}$-violating operator $\mathcal{O}_{\mathrm{CPV}} / \Lambda_{\mathrm{BSM}}^{2}$, coupled to QCD. Following an estimate similar to that in Eq. (1) one obtains

$$
\theta_{\mathrm{eff}}^{\mathrm{BSM}} \sim\left(\frac{\Lambda_{\chi}}{\Lambda_{\mathrm{BSM}}}\right)^{2} \sim 10^{-10}\left(\frac{100 \mathrm{TeV}}{\Lambda_{\mathrm{BSM}}}\right)^{2}
$$

which shows that the axion is not going to solve the strong $\mathrm{CP}$ problem in the presence of generic CP-violating new physics at the scale $\Lambda_{\mathrm{BSM}} \lesssim 100 \mathrm{TeV}$.

\section{CP-violating axion-nucleon couplings}

A remarkable consequence of $\theta_{\mathrm{eff}} \neq 0$ is the generation of a scalar axion coupling to nucleons, $g_{a N}^{S} a \bar{N} N$ (with $N=p, n$ ), which can be searched for in axion-mediated force experiments as suggested by Moody and Wilczek [7]. Focussing on the leading iso-spin singlet term (as in [7]), one has

$$
g_{a N}^{S} \simeq \frac{\theta_{\mathrm{eff}}}{f_{a}} \frac{m_{u} m_{d}}{m_{u}+m_{d}} \frac{\langle N|\bar{u} u+\bar{d} d| N\rangle}{2} \simeq 1.3 \cdot 10^{-12} \theta_{\mathrm{eff}}\left(\frac{10^{10} \mathrm{GeV}}{f_{a}}\right) .
$$

For the numerical evaluation we employed the value of the pion-nucleon sigma term $\sigma_{\pi N}=$ $\langle N|\bar{u} u+\bar{d} d| N\rangle\left(m_{u}+m_{d}\right) / 2=59.1 \pm 3.5 \mathrm{MeV}$ [8]. As pointed out in Ref. [9], a factor $1 / 2$ was missed in the original derivation of Moody and Wilczek [7].

Although Eq. (3) is sufficient for the sake of an estimate, it turns out to be conceptually unsatisfactory when we focus on the relevant question: how to properly impose the nEDM bound? The reason being the it misses extra contributions due to meson tadpoles $\left(\pi^{0}, \eta, \eta^{\prime}\right)$, which are generated by the same UV sources of CP violation responsible for $\theta_{\mathrm{eff}} \neq 0$ and are of the same size

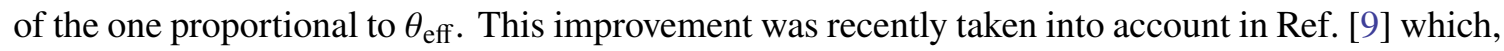
including as well iso-spin breaking effects from the leading order (LO) axion-baryon-meson chiral Lagrangian, found ${ }^{1}$

$$
\begin{aligned}
g_{a n, p}^{S} & \simeq \frac{4 B_{0} m_{u} m_{d}}{f_{a}\left(m_{u}+m_{d}\right)}\left[ \pm\left(b_{D}+b_{F}\right) \frac{\left\langle\pi^{0}\right\rangle}{F_{\pi}}+\frac{b_{D}-3 b_{F}}{\sqrt{3}} \frac{\left\langle\eta_{8}\right\rangle}{F_{\pi}}-\sqrt{\frac{2}{3}}\left(3 b_{0}+2 b_{D}\right) \frac{\left\langle\eta_{0}\right\rangle}{F_{\pi}}\right. \\
& \left.-\left(b_{0}+\left(b_{D}+b_{F}\right) \frac{m_{u, d}}{m_{d}+m_{u}}\right) \theta_{\mathrm{eff}}\right],
\end{aligned}
$$

where for clarity we neglected $m_{u, d} / m_{s}$ terms. Here, $B_{0}=m_{\pi}^{2} /\left(m_{d}+m_{u}\right)$ while the hadronic Lagrangian parameters $b_{D, F}$ are determined from the baryon octet mass splittings, $b_{D} \simeq 0.07 \mathrm{GeV}^{-1}$, $b_{F} \simeq-0.21 \mathrm{GeV}^{-1}$ at $\mathrm{LO}$ [12]. The value of $b_{0}$ is determined from the pion-nucleon sigma term as $b_{0} \simeq-\sigma_{\pi N} / 4 m_{\pi}^{2}$. From the determination in [8] one obtains $b_{0} \simeq-0.76 \pm 0.04 \mathrm{GeV}^{-1}$. Given $\sigma_{\pi N} \equiv\langle N|\bar{u} u+\bar{d} d| N\rangle\left(m_{u}+m_{d}\right) / 2$, the isospin symmetric $b_{0} \theta_{\text {eff }}$ term reproduces exactly Eq. (3).

In general, $g_{a N}^{S}$ and $d_{n}$ are not proportional, as it would follow instead from Eq. (3). For instance, exact cancellations among the VEVs can happen for $d_{n}[13,14]$ which have no counterpart in $g_{a N}^{S}$. Note that $\theta_{\mathrm{eff}}$ and the meson VEVs in Eq. (4) are meant to be computed from a highenergy source of $\mathrm{CP}$ violation, represented by an effective operator $\mathscr{O}_{\mathrm{CPV}}$. In Ref. [9] an explicit

\footnotetext{
${ }^{1}$ The importance of meson tadpole contributions was previously pointed out in [10], while iso-spin breaking effects were also taken into account in [11].
} 
example was worked out in the context of 4-quark operators of the type $\mathscr{O}_{\mathrm{CPV}}=(\bar{q} q)\left(\bar{q}^{\prime} i \gamma_{5} q^{\prime}\right)$ with $q, q^{\prime}=u, d, s$, arising e.g. by integrating out the heavy $W_{R}$ boson in left-right symmetric models. Building on the detailed analysis of Ref. [14], both $g_{a N}^{S}$ and $d_{n}$ were computed in the minimal left-right symmetric model with $\mathcal{P}$-parity, showing a non-trivial interplay which deviates sizeably from the naive approach of Eq. (3) with $g_{a N}^{S} \propto d_{n} \propto \theta_{\text {eff. }}$

\section{Axion-mediated forces}

Including both scalar and pseudo-scalar couplings to matter fields, the axion interaction Lagrangian can be written as ${ }^{2} \mathcal{L}_{a}^{\text {int }}=g_{a N}^{S} a \bar{N} N+g_{a f}^{P} a \bar{f} i \gamma_{5} f$ (here $f=N, e$ ), where $g_{a f}^{P}=C_{f} m_{f} / f_{a}$ is the usual pseudo-scalar axion coupling, with $C_{f} \sim O(1)$ in benchmark axion models [16]. By taking the non-relativistic limit of $\mathcal{L}_{a}^{\text {int }}$ one obtains different kinds of static potentials, which can manifest themselves as new axion-mediated macroscopic forces [7]. The latter can be tested in laboratory experiments, and hence do not rely on model-dependent axion production mechanisms, as in the case of dark matter axions (haloscopes) or to a less extent solar axions (helioscopes). An updated review of axion-mediated force experiments and relevant limits can be found in Ref. [17].

Axion-induced potentials can be of three types, depending on the combination of couplings involved, i.e. $g_{a N}^{S} g_{a f}^{S}$ (monopole-monopole), $g_{a N}^{S} g_{a f}^{P}$ (monopole-dipole) and $g_{a f}^{P} g_{a f}^{P}$ (dipoledipole). The idea of searching for dipole-dipole axion interactions in atomic physics is as old as the axion itself [3]. However, dipole-dipole forces turn out to be spin suppressed in the nonrelativistic limit and suffer from large backgrounds from ordinary magnetic forces. Searches based on monopole-monopole interactions, like tests of gravity on macroscopic scales, are in principle much more powerful. However, under the theoretical prejudice that we are after the QCD axion, the $\theta_{\text {eff }}^{2}$ suppression (given the nEDM bound) is such that current experiments are still some orders of magnitude far from testing the QCD axion.

The sweet spot is given by monopole-dipole searches which, as shown in Fig. 1, will enter the QCD axion region in the near future. In fact, a new detection concept by the ARIADNE collaboration $[18,19]$ plans to use nuclear magnetic resonance techniques to probe the axion field sourced by an unpolarized material via a sample of nucleon spins. Note that the yellow QCD axion band in Fig. 1 is obtained by employing Eq. (3) for the scalar axion-nucleon coupling, with the value of $\theta_{\text {eff }}$ spanning from the SM estimate $\theta_{\text {eff }} \sim 10^{-19}$ to the limit imposed by the nEDM $\theta_{\text {eff }} \simeq 10^{-10}$. However, as argued at the end of Sect. 3, the relation between $g_{a N}^{S}$ and $d_{n}$ is model dependent and hence, given a specific source of CP-violation, it should be assessed case by case in order to properly determine the parameter space region that is allowed by the nEDM bound.

\section{Outlook}

In order to have a testable signal in monopole-dipole axion searches a sizeable sources of $\mathrm{CP}$ violation beyond the SM is required. As a reference value in terms of $\theta_{\text {eff }}$ (using Eq. (3) for $g_{a N}^{S}$ ) one would need $\theta_{\mathrm{eff}} \gtrsim 10^{-13}$, that is three orders of magnitude below the current nEDM bound. Since CP is not a symmetry of nature, there is no reason to expect $\theta_{\text {eff }} \rightarrow 0$. The SM itself predicts $\theta_{\mathrm{eff}} \sim 10^{-19}$, that is far from being testable. However, new sources of CP violation beyond the CKM

\footnotetext{
${ }^{2}$ More general CP-violating axion-like particle interactions have been recently analyzed in [15].
} 


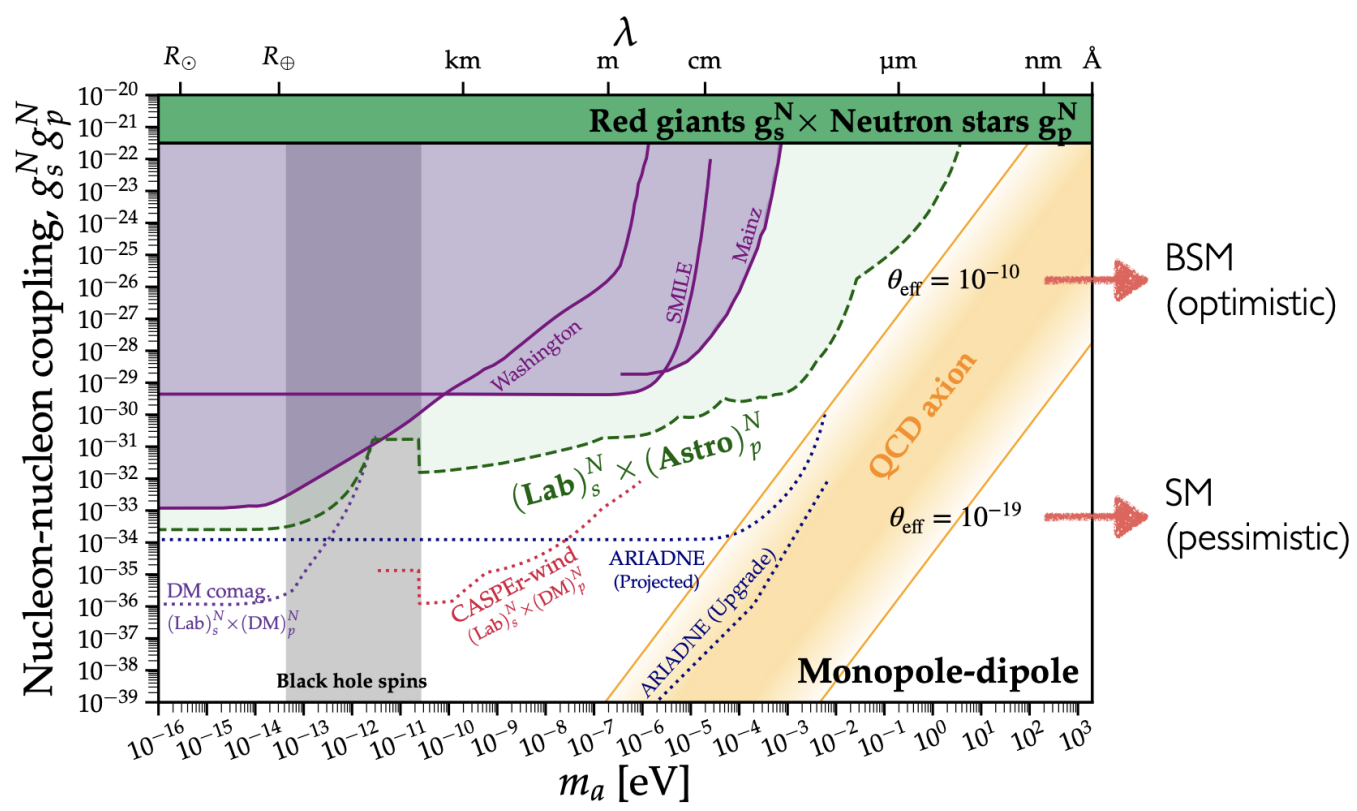

Figure 1: Parameter space of axion-mediated monopole-dipole forces. Figure from Ref. [17].

phase are needed to explain the matter-antimatter asymmetry of the universe and, even if decoupled at scales as heavy as $100 \mathrm{TeV}$, they might contribute sizeably to $\theta_{\text {eff }}$ (see estimate in Eq. (2)).

If an axion relaxation mechanism is at play, then axion-mediated forces provide an alternative experimental handle for probing UV sources of CP violation in the quark sector, with projected sensitivities that are stronger than current EDM searches. ${ }^{3}$ This suggests to rethink the role of the QCD axion from a "laundry detergent" of $\mathrm{CP}$ violation in the strong interactions to a low-energy portal to high-energy sources of $\mathrm{CP}$ violation, thus turning the strong $\mathrm{CP}$ problem into the strong CP opportunity.

\section{Acknowledgments}

I thank Stefano Bertolini, Giacomo Landini and Fabrizio Nesti for pleasant discussions and collaboration on the topics of this note.

\section{References}

[1] R.D. Peccei and H.R. Quinn, CP Conservation in the Presence of Instantons, Phys. Rev. Lett. 38 (1977) 1440.

[2] R.D. Peccei and H.R. Quinn, Constraints Imposed by CP Conservation in the Presence of Instantons, Phys. Rev. D16 (1977) 1791.

[3] S. Weinberg, A New Light Boson?, Phys. Rev. Lett. 40 (1978) 223.

\footnotetext{
${ }^{3}$ Future improvements on EDM limits might as well play a crucial role for disentangling different sources of $\mathrm{CP}$ violation coupled to QCD, also in the presence of a light axion field [20].
} 
[4] F. Wilczek, Problem of Strong $p$ and $t$ Invariance in the Presence of Instantons, Phys. Rev. Lett. 40 (1978) 279.

[5] C. Vafa and E. Witten, Parity Conservation in QCD, Phys. Rev. Lett. 53 (1984) 535.

[6] H. Georgi and L. Randall, Flavor Conserving CP Violation in Invisible Axion Models, Nucl. Phys. B276 (1986) 241.

[7] J.E. Moody and F. Wilczek, New Macroscopic Forces?, Phys. Rev. D30 (1984) 130.

[8] M. Hoferichter, J. Ruiz de Elvira, B. Kubis and U.-G. Meißner, High-Precision Determination of the Pion-Nucleon $\sigma$ Term from Roy-Steiner Equations, Phys. Rev. Lett. 115 (2015) 092301 [1506. 04142].

[9] S. Bertolini, L. Di Luzio and F. Nesti, Axion-mediated forces, CP violation and left-right interactions, Phys. Rev. Lett. 126 (2021) 081801 [2006. 12508].

[10] M. Pospelov, CP odd interaction of axion with matter, Phys. Rev. D58 (1998) 097703 [hep-ph/9707431].

[11] F. Bigazzi, A.L. Cotrone, M. Jarvinen and E. Kiritsis, Non-derivative Axionic Couplings to Nucleons at large and small N, 1906.12132.

[12] A. Pich and E. de Rafael, Strong CP violation in an effective chiral Lagrangian approach, Nucl. Phys. B367 (1991) 313.

[13] V. Cirigliano, W. Dekens, J. de Vries and E. Mereghetti, An $\epsilon^{\prime}$ improvement from right-handed currents, Phys. Lett. B 767 (2017) 1 [1612.03914].

[14] S. Bertolini, A. Maiezza and F. Nesti, Kaon CP violation and neutron EDM in the minimal left-right symmetric model, Phys. Rev. D 101 (2020) 035036 [1911.09472].

[15] L. Di Luzio, R. Gröber and P. Paradisi, Hunting for the CP violating ALP, 2010 13760.

[16] L. Di Luzio, M. Giannotti, E. Nardi and L. Visinelli, The landscape of QCD axion models, 2003.01100.

[17] C.A.J. O'Hare and E. Vitagliano, Cornering the axion with CP-violating interactions, Phys. Rev. D 102 (2020) 115026 [2010.03889].

[18] A. Arvanitaki and A.A. Geraci, Resonantly Detecting Axion-Mediated Forces with Nuclear Magnetic Resonance, Phys. Rev. Lett. 113 (2014) 161801 [1403. 1290].

[19] ARIADNE collaboration, Progress on the ARIADNE axion experiment, Springer Proc. Phys. 211 (2018) 151 [1710.05413].

[20] J. de Vries, P. Draper, K. Fuyuto, J. Kozaczuk and B. Lillard, Uncovering an Axion Mechanism with the EDM Portfolio, 2107.04046. 